\title{
La investigación formativa como herramienta para la innovación educativa
}

\section{Formative research as a tool for educational innovation}

\author{
Edgar Rigoberto Curay Banegas ${ }^{1 *}$ y Oscar Santiago Vanegas Quizhpi ${ }^{1}$ \\ ${ }^{1}$ Universidad Católica de Cuenca \\ *ecuray@ucacue.edu.ec
}

DOI: https://doi.org/10.26871/killkana_social.v2i2.313

\begin{abstract}
Resumen
En la actualidad, la investigación formativa en Educación Superior sitúa al estudiante como protagonista principal de la generación de su propio conocimiento. La función de los docentes está orientada a utilizar métodos adecuados que permitan: tutorar, guiar, asistir y acompañar a los estudiantes hacia la consecución de un aprendizaje significativo e independiente, motivándolos a trabajar de manera colaborativa y autónoma según el caso. En el siguiente artículo se reflexiona sobre la experiencia derivada de la investigación formativa en la Unidad Académica de Educación de la Universidad Católica de Cuenca (UCACUE), donde se tiene por objetivo la innovación educativa al promover de forma permanente y sistemática la formación de investigadores a nivel de pregrado, con énfasis en el aprender haciendo. La finalidad del estudio que se presenta es el análisis de la innovación a través del proyecto integrador en las carreras de Educación Inicial y Sicología Educativa de la Universidad Católica de Cuenca. Los principales resultados obtenidos evidencian la idoneidad de la innovación docente implementada para potenciar el desarrollo de habilidades de pensamiento de orden superior. El desarrollo de estas competencias se logró gracias principalmente al desarrollo de la investigación formativa como trabajo integrador, lo cual permitió generar productos académicos que han sido expuestos en la modalidad de posters científicos, evaluados por los docentes en la casa abierta de fin de ciclo.
\end{abstract}

Palabras clave: investigación, formativa, pregrado, innovación, nueva educación.

\begin{abstract}
At present, the formative research in higher education places the student as the main protagonist of the generation of his own knowledge. The role of teachers is oriented to use appropriate methods that allow: tutorate, guide, assist and accompany students towards the achievement of meaningful and independent learning, motivating them to work collaboratively and independently depending on the case. In the following article we reflect on the experience derived from formative research in the Academic Unit of Education of the Catholic University of Cuenca (UCACUE) having as objective the educational innovation to promote in a permanent and systematic way the formation of researchers at the undergraduate level emphasizing learning by doing.The purpose of the study we present is the analysis of innovation through the integrative project in the careers of Initial Education and Educational Psychology of the Catholic University of Cuenca, the main results obtained being evidence of the suitability of the teaching innovation implemented to enhance the development of higher order thinking skills. The development of these competences has been achieved thanks mainly to the development of formative research as integrative work, which has allowed the generation of academic products, which have been exposed in the form of scientific posters, being evaluated by teachers in the open house of end of cycle.
\end{abstract}

Key words: research, training, undergraduate, innovation, new education.

\section{Introducción}

La Educación Superior del Ecuador desde hace más de una década se encuentra viviendo una serie de innovaciones de carácter profundo, con la premisa de la constante búsqueda de la excelencia académica. Esta innovación educativa a nivel superior puede darse desde diversos ámbitos: procesos de inclusión, uso de metodologías interactivas, programas de fomento de la investigación.
En relación a los procesos de inclusión educativa en la educación superior, el Reglamento de la LOES (2012), en su artículo 229 se refiere a que las instituciones brindarán atención de estudiantes con necesidades educativas especiales [NEE] y que contarán con equipos de profesionales especializados en su detección, quienes además deberán definir cuál es la modalidad más adecuada para cada estudiante y la atención complementaria, por lo que la creación de dicha unidad para la detección de NEE tiene el propósito de brindar las mejores oportunidades a 
los estudiantes incluidos, para disponer de un espacio en donde sean evaluados de manera integral desde la perspectiva psicopedagógica inclusiva, con vistas a desarrollar competencias y habilidades que les permitan incluirse en los procesos educativos ordinarios o, de ser requerido, en procesos educativos especializados.

Para hacer efectiva la inclusión educativa es necesario diseñar e implementar las adaptaciones curriculares que permiten afrontar las particularidades de la relación entre el estudiante y su ambiente. Es así como el Reglamento de Régimen Académico [RRA] (2013), en sus artículos 40 y 53 considera algunos aspectos del proceso de enseñanza que hacen posible una educación universitaria inclusiva, como son: educación con recursos adaptados, medios y ambientes de aprendizaje apropiados, uso de ambientes y metodologías de aprendizaje; y contenidos curriculares que respeten y potencien las capacidades diversas (Herdoiza, 2015).

Según la propuesta del Reglamento del Sistema de Evaluación Estudiantil [RSEE] (CES 2017), en su artículo 15 sobre la Evaluación de los aprendizajes de estudiantes con necesidades especiales se puntualiza que "las IES deberán desarrollar políticas, programas y planes de acción afirmativa e inclusión educativa, en los cuales habrán de contemplarse metodologías, ambientes de aprendizajeenseñanza, instrumentos y baterías de evaluación que propendan al aprendizaje universal".

Para hacer efectiva la inclusión de los estudiantes en el sistema educativo es necesario en algunas ocasiones realizar las adaptaciones curriculares que son entendidas "como un conjunto de medidas que se toman para un determinado alumno, pero con una perspectiva sistémica, global, de centro.” (Ruiz Rodríguez, E. , 2003)

Con lo que respecta a la implementación de una metodología interactiva en la educación superior, esta es una labor diaria que se desarrolla a nivel mundial, en Latinoamérica y en el Ecuador. Es variada la metodología interactiva que se trabaja en distintos lugares, así lo confirma Samwel (2010) quien realizó un trabajo de campo revisando 21 artículos, identificando 26 métodos distintos, de los cuales los más utilizados eran conferencias y teorías, estudio de casos, debates y trabajo de grupo, simulación de empresas, videos y películas, invitados, creación de planes de negocio, proyectos, creación de empresas reales, juegos y competencias, visitas, presentaciones, workshops, presentaciones y visitas, aprendizaje basado en problemas, aprendizaje basado en proyectos, entre otros

En relación a los programas de fomento de la investigación a nivel superior, que es el motivo de este artículo, el Ecuador encuentra su punto de partida en la idea de cambio de matriz productiva del país, el mismo que plantea el potenciar la formación de profesionales con capacidades investigativas como valor agregado a nivel de las instituciones de educación superior. Esta tarea resulta ser de capital importancia para sostener el proceso mismo de cambio de matriz productiva y, dado que implica un giro conceptual, vuelve a su implantación en algo muy complejo.

La Ley Orgánica de Educación Superior [LOES] proporciona el marco legal para esta revolución académica e investigativa. Así, en su artículo 8, entre los fines de la educación superior está el "f) fomentar y ejecutar programas de investigación de carácter científico, tecnológico y pedagógico que coadyuven al mejoramiento y protección del ambiente y promuevan el desarrollo sustentable nacional" (Asamblea Nacional del Ecuador, 2010).

El artículo 13 de esta misma ley versa sobre las funciones de la educación superior entre las que se destacan:

a) Garantizar el derecho a la educación superior mediante la docencia, la investigación y su vinculación con la sociedad, y asegurar crecientes niveles de calidad, excelencia académica y pertinencia; [...] d) Fortalecer el ejercicio y desarrollo de la docencia y la investigación científica en todos los niveles y modalidades del sistema; [...] k) Promover mecanismos asociativos con otras instituciones de educación superior, así como con unidades académicas de otros países, para el estudio, análisis, investigación y planteamiento de soluciones de problemas nacionales, regionales, continentales y mundiales; [...] ñ) Brindar niveles óptimos de calidad en la formación y en la investigación (Asamblea Nacional del Ecuador, 2010).

La investigación científica tiene como objetivo explicar los fenómenos para derivar predicciones contrastables sobre la realidad, construyendo teorías que relacionen consistentemente los fenómenos (Feynman, R. P., 1994). A partir de los datos obtenidos se pueden generar programas de vinculación con la sociedad y la información obtenida de estos dos procesos puede utilizarse en el aula para reafirmar los contenidos científicos expuestos con las experiencias de campo.

La Universidad Católica de Cuenca [UCACUE] en armonía con las políticas públicas antes enunciadas y a través de su Unidad Académica de Educación le correspondía crear y desarrollar metodologías curriculares compatibles con las exigencias de la sociedad actual. Con la idea de contrarrestar los resultados de un enfoque tradicional en la formación de investigadores, a pesar de contar en los currículos con asignaturas relacionadas a la investigación, se observaba que los estudiantes llegaran hasta su titulación sin haber alcanzado los estándares mínimos en competencias investigativas, e incluso en donde el currículo mismo se convierte en el primer obstáculo para el desarrollo de los semilleros (Quintero, Munévar, y Munévar, 2008).

Implementar la investigación formativa mediante la ejecución del proyecto integrador a través de una asignatura en cada ciclo, favorece la articulación entre la investigación formativa con la científica desde el inicio del pregrado. La implementación de esta estrategia se la trabaja de manera transversal al currículo y consustancial a una de las grandes vertientes o estrategias de enseñanza: la de aprendizaje por descubrimiento. Así, se busca una construcción del conoci- 
miento desde la práctica profesional, con la investigación como proceso y resultado principal.

Así, se conceptualiza investigar como una parte integral del proceso de formación de los estudiantes, como un estilo de vida, un verdadero saber haciendo, donde "la práctica y el ejercicio van implícitos en la adquisición de una capacidad". (Dewey, 1993).

\section{Metodología}

La metodología empleada por todos los grupos estudiantiles que participaron en este proyecto fue el aprendizaje por descubrimiento, donde se planteo como objetivo que sea el propio alumno el que adquiere una gran parte de los conocimientos por sí mismo, a través de su experiencia personal de descubrimiento. Al inicio del periodo académico, los docentes de cada nivel consensuan y distribuyen la temática a investigar, y los estudiantes presentan sus avances a los docentes tutores. Al concluir el periodo académico se presentan los resultados finales, en soporte digital, escrito (paper) y una exposición oral, los mismos que son evaluados por un jurado de docentes. Así, el docente actúa como guía o mediador, los estudiantes son los que generan el producto investigativo, limitándose el docente a presentar todas las herramientas necesarias para que este descubra de un modo personal y autónomo lo que desea aprender.

\section{Desarrollo}

Se ha manifestado que la innovación educativa a nivel superior puede darse desde diversas aristas: procesos de inclusión de estudiantes con necesidades educativas especiales asociadas o no a una discapacidad, uso de metodologías interactivas a los procesos de interaprendizaje, en donde no solo los estudiantes aprenden, sino también los docentes mediante la interacción, o implementación de programas de investigación formativa.

La reflexión en este artículo se va a realizar entorno a los procesos de investigación formativa, ya que este se ha constituido en un elemento vital del quehacer universitario, se dice que una universidad que no investiga no crece, o que la investigación se ha convertido en la mejor amiga de la verdad, por lo que es necesario pensar en una innovación educativa desde la investigación que posibilite el desarrollo de competencias adecuadas que viabilizará un desempeño eficaz en el ejercicio profesional.

Según Restrepo (2003):

La finalidad de este programa de innovación educativa mediante la investigación formativa es difundir información existente y favorecer que el estudiante la incorpore como conocimiento, es decir, desarrolla las capacidades necesarias para el aprendizaje permanente, necesario para la actualización del conocimiento y el desarrollo de habilidades y competencias profesionales. (p.196)

La investigación formativa se convierte en un verdadero semillero (sitio donde siembra o se va a trasplantar), constituyéndose en el lugar o espacio que ofrece las condiciones iniciales para su germinación (Llamas, 2015).
La investigación formativa, anclada al proyecto integrador, nace como un Programa de Fomento a la Investigación Educativa en la Unidad Académica de Educación, Artes y Humanidades, en las Sedes y Extensiones por la necesidad de impulsar y desarrollar la investigación educativa como eje integrador, lo cual permite que los estudiantes interioricen los procesos investigativos en la experiencia del aprender haciendo, y que dicho proyecto se convierta en una plataforma para destacar a los profesionales en formación y sus proyectos.

Como objetivo general se planteó:

Promover la formación de actitudes y competencias básicas investigativas de los estudiantes de pregrado, de forma permanente y sistemática, a fin de que se apropien de los conocimientos trabajados, recapaciten, experimenten y aporten mediante la investigación a lo pedagógico, a la transformación del conocimiento y a las comunidades con las que trabajan, favoreciendo así un mayor desarrollo y mejor calidad de vida de las personas con las que se relacionan. (Curay, 2015).

Mientras que los objetivos específicos son:

1) Favorecer a docentes y estudiantes investigadores de herramientas teóricos metodológicas que les permitan desarrollar proyectos de investigación educativa, divulgar sus resultados e impacto en las propuestas de innovación educativa, con el propósito de interrelacionar la docencia con la investigación y la vinculación con la sociedad.

2) Promover la investigación educativa, como actividad básica institucional de Unidad Académica de Educación Artes y Humanidades, elevando el nivel cualitativo y la relevancia de las actividades de investigación y la transferencia del nuevo conocimiento.

3) Ampliar de manera sustancial el número de publicaciones de las investigaciones en textos, libros y revistas indexadas de alto impacto, la presentación de resultados de investigación en eventos de trascendencia, y la organización de certámenes que conduzcan a la divulgación y confrontación de nuevos conocimientos.

4) Guiar las acciones de la investigación hacia un enfoque basado en la búsqueda de la calidad intelectual, la relevancia temática de sus aportes y un enfoque sistémico, sustentable e interdisciplinario que refleje la misión y visión de la Universidad.

5) Diseñar e implementar cursos-talleres para la formación de profesores en investigación educativa para que luego sean replicados a los estudiantes.

6) Formar redes de estudiantes y docentes investigadores en educación.

7) Articular los procesos de investigación, vinculación con la sociedad y la docencia. (Curay, 2015).

Un punto crucial para el desarrollo de los trabajos fue la detección de problemas socioeducativos (desde preparatoria hasta universitario) por parte de los estudiantes en los sitios donde realizan sus prácticas. Posterior a la detección de dichos problemas, por medio de debates académicos 
dentro de su cátedra integradora, se seleccionan los temas para desarrollar el respectivo proyecto de investigación.

Cabe destacar la importancia del docente responsable de la cátedra integradora, encargado de la recepción de las temáticas a tratar, de supervisar la conformación de los grupos de estudiantes, de la socialización de los cronogramas para el cumplimiento, de coordinar el trabajo de campo y de presidir los jurados que se encargarán de receptar las defensas de los proyectos. Dicho docente cuenta con el apoyo de sus pares académicos en calidad de tutores individuales de cada grupo y, de ser necesaria, de su experticia individual en los distintos campos del conocimiento.

Entre las consideraciones más importantes al realizar los trabajos de investigación se tiene que: a) los temas escogidos deben ajustarse a las líneas de investigación determinadas en cada carrera, b) en los proyectos en que se integran diferentes asignaturas, cada una de ellas aporta un objetivo específico o resultado de aprendizaje, y es la cátedra integradora la encargada de coordinar las actividades del desarrollo y evaluación del proceso investigativo; y, c) la investigación formativa de los estudiantes de los últimos ciclos se enfoca exclusivamente en su investigación de fin de carrera.

La puntuación de evaluación de la investigación formativa en cada asignatura obligatoriamente será de 15 puntos dentro de la nota total del aprovechamiento. El puntaje otorgado a la investigación formativa se atribuye en base a los siguientes aspectos: a) la entrega del reporte final de investigación formativa será requisito para presentar la defensa del proyecto y será verificado por el docente responsable de la cátedra integradora de cada nivel de formación y avalado por el Director de carrera, b) se deberá garantizar la originalidad del trabajo a través del software Turnitin, con una tolerancia máxima del 5\%. (Consejo Universitario de la Universidad Católica de Cuenca, 2016).

Los escenarios en los cuales se desarrollan las investigaciones son instituciones educativas de preparatoria, básica elemental, básica superior, bachillerato y de educación superior del cantón Cuenca - Ecuador, donde los estudiantes realizan sus prácticas pre profesionales. Los eventos de exposición de resultados se llevan a cabo en diversos espacios públicos de la ciudad con el libre acceso del público en general.

Un aspecto importante a discutir en relación a los semilleros de investigación vividos es el hecho de haber trabajado de manera coordinada, articulada e interdisciplinariamente entre docentes y estudiantes, lográndose a más de llevar a buen puesto el proyecto, aumentar la producción académica la cual ha sido presentada en eventos científicos.

Tabla 1. Productos de los procesos de investigación formativa presentados en ponencias hasta la fecha.

\begin{tabular}{|c|c|}
\hline Evento & Título de la ponencia \\
\hline $\begin{array}{l}\text { III Congreso } \\
\text { internacional } \\
\text { de ciencias } \\
\text { pedagógicas. ITB, } \\
\text { Guayaquil. } 2017\end{array}$ & $\begin{array}{c}\text { Inclusión educativa y la Enseñanza de Lenguas } \\
\text { basada en problemas }\end{array}$ \\
\hline
\end{tabular}

\begin{tabular}{|c|c|}
\hline Evento & Título de la ponencia \\
\hline $\begin{array}{l}\text { III Congreso } \\
\text { internacional } \\
\text { de ciencias } \\
\text { pedagógicas. ITB, } \\
\text { Guayaquil. } 2017\end{array}$ & $\begin{array}{c}\text { Inclusión educativa y rendimiento académica. } \\
\text { Prueba FF-SIL para la detección de } \\
\text { disfuncionalidad familiar }\end{array}$ \\
\hline $\begin{array}{l}\text { III Congreso } \\
\text { internacional } \\
\text { de ciencias } \\
\text { pedagógicas. ITB, } \\
\text { Guayaquil. } 2017\end{array}$ & $\begin{array}{l}\text { Comunidades de aprendizaje como herramientas } \\
\text { de inclusión. Proyecto El círculo del Piano" }\end{array}$ \\
\hline $\begin{array}{l}\text { IV Reunión cientíi- } \\
\text { ca: investigación pa- } \\
\text { ra la innovación. IN- } \\
\text { PIN } 2017\end{array}$ & $\begin{array}{c}\text { La investigación formativa de pregrado en la } \\
\text { Universidad de Cuenca. Los semilleros de } \\
\text { investigación }\end{array}$ \\
\hline $\begin{array}{l}\text { T Congreso } \\
\text { internacional } \\
\text { de ciencias } \\
\text { pedagógicas. ITB, } \\
\text { Guayaquil. 2015 }\end{array}$ & $\begin{array}{l}\text { Barreras para el acceso a la Universidad de las } \\
\text { personas con discapacidad en Ecuador }\end{array}$ \\
\hline $\begin{array}{l}\text { II Congreso } \\
\text { internacional } \\
\text { de ciencias } \\
\text { pedagógicas. ITB, } \\
\text { Guayaquil. } 2016\end{array}$ & $\begin{array}{l}\text { Incremento de la carga horaria en Educación } \\
\text { Física sobre el estado nutricional de los escolares }\end{array}$ \\
\hline $\begin{array}{l}\text { II Congreso } \\
\text { internacional } \\
\text { de ciencias } \\
\text { pedagógicas. ITB, } \\
\text { Guayaquil. 2016 }\end{array}$ & $\begin{array}{c}\text { Desarrollo de la unidad de diagnóstico, } \\
\text { investigación psicopedagógica y de apoyo a la } \\
\text { inclusión (UDIPSAI-UCACUE) para la atención } \\
\text { de estudiantes con necesidades educativas } \\
\text { especiales asociadas o no a la discapacidad }\end{array}$ \\
\hline $\begin{array}{l}\text { II Congreso } \\
\text { internacional } \\
\text { de ciencias } \\
\text { pedagógicas. ITB, } \\
\text { Guayaquil. 2016 }\end{array}$ & $\begin{array}{c}\text { La discapacidad intelectual y el aprendizaje de la } \\
\text { lectoescritura }\end{array}$ \\
\hline $\begin{array}{lr}\text { TX } & \text { Encuentro } \\
\text { internacional } & \text { de } \\
\text { estudiantes } & \text { de } \\
\text { psicología } & \end{array}$ & $\begin{array}{c}\text { La evaluación psicopedagógica y la inclusión } \\
\text { educativa }\end{array}$ \\
\hline $\begin{array}{lr}\text { IX } & \text { Encuentro } \\
\text { internacional } & \text { de } \\
\text { estudiantes } & \text { de } \\
\text { psicología } & \end{array}$ & Dolor de espalda y mochila escolar \\
\hline $\begin{array}{lr}\text { IX Encuentro } \\
\text { internacional } & \text { de } \\
\text { estudiantes } & \text { de } \\
\text { psicología } & \end{array}$ & $\begin{array}{c}\text { Evaluación de un programa para el desarrollo del } \\
\text { pensamiento lógico formal de estudiantes de } \\
\text { décimo grado de Educación Básica, en Cuenca, } \\
\text { Ecuador }\end{array}$ \\
\hline
\end{tabular}

Tabla 2. Productos de los Semilleros de Investigación publicados en revistas indexadas hasta la fecha

\begin{tabular}{|l|c|}
\hline Publicación & Título del artículo \\
\hline $\begin{array}{l}\text { Revista de ciencias } \\
\text { pedagógicas e Inno- } \\
\text { vación. UPSE. vol } \\
\text { 5. núm 3. 2017 }\end{array}$ & $\begin{array}{c}\text { Information-gap activities en la comprensión } \\
\text { lectora de textos en inglés }\end{array}$ \\
\hline $\begin{array}{l}\text { Revista cubana de } \\
\text { Medicina militar. } \\
\text { vol 45. núm 4. 2016 }\end{array}$ & $\begin{array}{c}\text { Impacto en el aumento del ejercicio físico sobre el } \\
\text { estado nutricional de los escolares }\end{array}$ \\
\hline $\begin{array}{l}\text { Revista de Psicolo- } \\
\text { gía Iztacala. vol 20. } \\
\text { núm 3. 2017 }\end{array}$ & $\begin{array}{c}\text { Adolescentes de básica superior con adicción a } \\
\text { internet y redes sociales y relaciones } \\
\text { interpersonales }\end{array}$ \\
\hline $\begin{array}{l}\text { Revista de Psicolo- } \\
\text { gía Iztacala. vol 20. } \\
\text { núm 3. 2017 }\end{array}$ & $\begin{array}{c}\text { Creencias, actitudes y prácticas en sexualidad en } \\
\text { estudiantes de psicología clínica de la universidad } \\
\text { católica de cuenca }\end{array}$ \\
\hline $\begin{array}{l}\text { Revista IPLAC. no } \\
\text { 3.2015. sección: ex- } \\
\text { periencias educati- } \\
\text { vas. }\end{array}$ & $\begin{array}{c}\text { Acercamiento necesario al mejoramiento de la } \\
\text { práctica docente de los profesores universitarios. }\end{array}$ \\
\hline $\begin{array}{l}\text { Revista dilemas } \\
\text { contemporáneos: } \\
\text { educación, política } \\
\text { y valores. Año 4. } \\
\text { núm 2. 2017 }\end{array}$ & $\begin{array}{l}\text { La práctica de valores y su incidencia en la } \\
\text { convivencia escolar. }\end{array}$ \\
\hline
\end{tabular}

\section{Conclusiones}

Se ha logrado un proceso de investigación formativa como eje fundamental para la formación para la investigación y para la formación de profesionales con pensamiento crítico, con capacidad para el aprendizaje permanente, de 
búsqueda de problemas no resueltos y de plantear soluciones en su labor cotidiana, características del tipo de profesionales que requiere el país. Particularmente el proyecto Semillero de Investigación ha contribuido con varios aportes tanto para la institución como para sus participantes.

Entre los beneficios obtenidos para la institución se $\log \operatorname{rar}:$ :

- Incremento de los indicadores de acreditación de la Unidad Académica en producción académica.

- Apoyo a la investigación docente institucional e interinstitucional.

- Difusión de las producciones de la unidad académica ante la comunidad científica.

- Promoción de las carreras ante la comunidad y el aumento de las propuestas de vinculación universitaria por parte de los ciudadanos.

- Apropiación de nuevos lenguajes que fortalecen el diálogo entre el saber pedagógico, el saber disciplinar, el saber investigativo y los productos de la ciencia y la tecnología.

Entre los beneficios para los estudiantes están:

- Abordaje de las tesis y trabajos de grado con mayor seguridad y madurez intelectual.

- Iniciarse como investigadores.

- Recibir certificaciones institucionales para la hoja de vida como futuro profesional o para aplicar a becas, convocatorias nacionales e internacionales, pasantías y demás estímulos académico-científicos.

- Familiarizarse desde etapas tempranas con las diferentes formas de investigar y producción de conocimiento.

- Mayor seguridad cuando llegan a la etapa final próxima a recibir el título y la inserción en los contextos reales de práctica educativa.

- Intercambiar experiencias con pares de otras áreas y otras instituciones.

Para concluir, se enumeran las necesidades a corto plazo que se requieren para el avance de este proyecto.

- Elaborar un código de ética para proyectos de investigación formativa.

- Estructurar, atender y dimensionar la documentación y archivo pedagógico de los proyectos y de los grupos.

- Participar por docente, como mínimo, en un congreso o evento científico durante el año.

- Hacer las relatorías, protocolos y actas de las sesiones, seminarios y eventos con el fin de dejar memoria escrita para las bases de datos institucionales.

\section{Referencias Bibliográficas}

Asamblea Nacional del Ecuador. (2010). Ley Orgánica de Educación Superior [LOES].
Consejo Universitario de la Universidad Católica de Cuenca. (2016). Manual de investigación formativa. Descargado de https://onedrive.live.com/ ?authkey=21ANG 84 ZuCWos $65 \mathrm{ug} \& \mathrm{cid}=$ FCB80E4324F0D639\&id= FCB80E4324F0D639\%21795\&parId= FCB80E4324F 0D 639\%21659\&O=OneUp

Curay, E. (2015). Propuesta de investigación educativa. Unidad Académica de Educación, Artes y Humanidades.

Dewey, J. (1993). Cómo pensamos. Nueva exposición de la relación entre pensamiento y proceso educativo. Barcelona, España: Paidós.

Feynman, R. P. (1994). The character of physical law. New York: Modern library.

Herdoiza, M. (2015). Costruyendo igualdad en la Educación Superior. Quito: Senescyt/Unesco.

Llamas, J. (2015). Importancia de los semilleros de investigación en la Universidad de Cartagena. Revista Palobra, 7(7), 137-141. Descargado de http: //revistas.unicartagena.edu .co/index.php/palobra/article/view/ $159 / 124$

Quintero, J., Munévar, R., y Munévar, F. (2008). Semilleros de investigación: una estrategia para la formación de investigadores. Educación y Educadores, 11(1), 31-42. Descargado de http://www.redalyc . org/pdf/834/83411103.pdf

Restrepo Gómez, B. . (2003). Investigación formativa e investigación productiva de conocimiento en la universidad. Nómadas, 18, 195-202.

Ruiz Rodríguez, E. . (2003). Adaptaciones curriculares individuales para los alumnos con Síndrome de Down. Revista Síndrome de Down, 4-15.

Recibido: 18 de junio de 2018

Aceptado: 29 de junio de 2018 
